\title{
The "Journal of Functional Morphology and Kinesiology" Journal Club Series: Highlights on Recent Papers in Musculoskeletal Disorders
}

\author{
Marta Anna Szychlinska ${ }^{1, *}$, Kotaro Yamakado ${ }^{2}$, Alessandro Castorina ${ }^{3}$ and \\ Milos Ljubisavljevic ${ }^{4}$ \\ 1 Department of Biomedical and Biotechnological Sciences, Human Anatomy and Histology Section, \\ School of Medicine, University of Catania, 95123 Catania, Italy \\ 2 Department of Orthopaedics/Sports Medicine, Fukui General Hospital, 9108561 Fukui, Japan; \\ yamakadok@gmail.com \\ 3 School of Life Sciences, Faculty of Science, University of Technology Sydney, PO Box 123, Broadway, \\ Sydney, New South Wales 2007, Australia; Alessandro.Castorina@uts.edu.au \\ 4 Department of Physiology, College of Medicine and Health Sciences, United Arab Emirates University, \\ 15551 Al-Ain, UAE; milos@uaeu.ac.ae \\ * Correspondence: marta.sz@hotmail.it
}

Received: 14 April 2017; Accepted: 14 April 2017; Published: 18 April 2017

\begin{abstract}
We are glad to introduce the fourth Journal Club. This edition is focused on several relevant studies published in the last few years in the field of musculoskeletal disorders, chosen by our Editorial Board members. We hope to stimulate your curiosity in this field. The Editorial Board members wish you an inspiring lecture.
\end{abstract}

Keywords: musculoskeletal disorders; osteoarthritis; Mediterranean diet; rotator cuff tears; fibromyalgia

\section{Introduction}

Musculoskeletal disorders (MSDs) are pathological conditions that affect muscles, bones, ligaments, tendons, nerves, and joints. MSDs can affect any major area of your musculoskeletal system, such as neck, shoulders, wrists, back, hips, legs, knees, and feet. They include conditions such as: tendinitis, carpal tunnel syndrome, osteoarthritis, rotator cuff tear, rheumatoid arthritis, fibromyalgia, bone fractures, and others. MSDs are one of the most common causes of pain and disability throughout the world. Aging, obesity, excessive mechanical stress, acute injuries, awkward posture, repetitive movements, and metabolic disorders are considered among the most prominent risk factors [1-3]. The severity of MSDs can vary. In some cases, they cause pain and discomfort that interferes with everyday activities. Nowadays, no resolving cure exists for the majority of MSDs, thus, early diagnosis and treatment are of fundamental importance in avoiding disability and improving the long-term outlook. It is very important to be able to recognize the major symptoms of MSDs, which can include: recurrent pain, stiff joints, swelling, and dull aches [4]. MSDs are, currently, extensively reported in literature. Many different preventive and therapeutic approaches are taken into account such as nutrition, including natural dietary supplements use, physical activity protocols, tissue reconstruction, tissue engineering, and many others. MSDs are becoming increasingly common worldwide and the pathophysiological mechanisms that trigger their development and progression need to be further studied. In the sections that follow, some interesting papers are reported on in an effort by our editorial board members to highlight some novel preventive, therapeutic, and diagnostic approaches for the most common MSDs. 


\section{Recent Papers Regarding Musculoskeletal Disorders}

\subsection{Effects of Mediterranean Diet on Osteoarthritis Onset}

Highlight by Marta Anna Szychlinska

One of the most common causes of disability, especially in elderly populations, are chronic musculoskeletal disorders. Osteoarthritis (OA) represents the most common musculoskeletal pathologic condition and is estimated to be the 11th highest contributor to worldwide disability [5]. Mediterranean Diet (MD) refers to the traditional dietary habits of the Mediterranean region and is usually referred to as a cultural model for healthy eating [6]. The benefits of this diet, in relation to reducing the risk of developing musculoskeletal, cardiovascular, metabolic, and cognitive diseases, have been widely studied. Nevertheless, its effects on OA onset have only recently started to be investigated. Indeed, there are only a few papers investigating the effect of MD on OA. In a very interesting study by Musumeci et al., for example, it is reported that the consumption of Sicilian extra virgin olive oil, an essential component of MD, in conjugation with regular physical activity, are associated with the reduction of the pro-inflammatory cytokines expression (in particular interleukin-1), and the increased expression of lubricin, a chondroprotective glycoprotein with lubricating properties [7]. Moreover, recently, it has been shown that the adherence to a MD is associated with a lower prevalence of knee OA in a large cohort from North America [8]. In this remarkable and large cross-sectional study by Veronese et al., including 4358 participants with a mean age of 61 years, almost $30 \%$ of the cohort presented knee OA, diagnosed both clinically and radiologically. Adherence to the MD was evaluated using a MD score based on a food frequency questionnaire. The latter took into consideration several foods commonly consumed within the MD such as cereals, potatoes, fruits, vegetables, legumes, fish, red meat, poultry, full fat dairy products (e.g., milk cheese, yogurt), and alcoholic beverages. The frequency of consumption was reported at nine levels of intake from "never" to "every day". In addition, seven dietary behaviour questions were available regarding food preparation methods and fat intake, one question on fibre intake, and 13 questions on vitamin and mineral intakes. Some additional covariates such as body mass index (BMI), physical activity, race, smoking habit, educational attainment level, yearly income, and self-reported comorbidities were also evaluated. The results clearly indicated that participants with the highest adherence to MD had a significantly reduced probability of knee OA. Other factors significantly associated with knee OA were: BMI, non-white ethnicity, and college level education, while age was marginally significant. These data suggest a protective role for some components of MD, most of which are reported to have anti-inflammatory and antioxidant properties and to be good sources of vitamins and minerals, confirming its benefits on health outcomes.

\subsection{Arthroscopic Superior Capsule Reconstruction for Irreparable Rotator Cuff Tears}

Highlight by Kotaro Yamakado

The treatment of massive irreparable rotator cuff tears presents a significant challenge. There are many surgical options, ranging from arthroscopic debridement concomitant with biceps tenotomy, partial repair of the rotator cuff, patch grafts, muscle transfers, and reverse total shoulder arthroplasty (RSA). However, reported outcomes of these procedures are mixed and some of them are with a significant risk of complications.

Superior capsular reconstruction (SCR) was originally described by Mihata et al. in 2013 with a promising outlook [9]. Many shoulder surgeons, though quite suspicious at first, began to increasingly believe in its potential. In the original description of SCR, autograft fascia lata was harvested from the patient's thigh. As an alternative idea, acellular dermal allograft has been used [10-12]. The graft is attached medially to the superior glenoid and laterally to the greater tuberosity, arthroscopically. The biomechanical concept of SCR is to stabilize the glenohumeral joint from superior translation that occurs with a rotator cuff deficient shoulder. 
Mihata et al. published good short-term results [9]. They demonstrated clinical outcomes of 23 patients undergoing SCR with a fascia lata autograft. After a minimum follow-up period of 2 years, all clinical outcomes scores significantly improved: the American Shoulder and Elbow Surgeons score improved from 23.5 preoperatively to 92.9 postoperatively, and muscle strength significantly improved as well. Subsequently, Hirahara et al. reported technical feasibility of acellular dermal allograft usage [10]. Burkhart et al. identified advantages of SCR over reverse shoulder arthroplasty: (very) low infection rate, lower cost, and low morbidity [12]. They also stated that SCR does not preclude further RSA. From the biomechanical standpoint, Mihata et al. tested subacromial peak contact pressure, glenohumeral superior translation, and glenohumeral compression force using cadaveric shoulders, showing that SCR completely restored the superior stability of the shoulder joint by establishing posterior continuity between the graft, residual infraspinatus tendon, and underlying shoulder capsule [13].

SCRs are a very appealing option. Further study (long-term clinical and/or biomechanical) are anticipated.

\subsection{Work-Related Musculoskeletal Disorders in Office-Based Workplaces}

Highlight by Alessandro Castorina

With the advance of technology and the growing necessity of rapid communication, the work environment for skilled professionals such as accountants, lawyers, architects, academics, and engineers has changed in modern times, especially with the computerization of their office workplace [14]. The increasing use of computers in the workplace has also led to the introduction of a specific category of work-related musculoskeletal disorders (MSDs) for office-based workers. MSDs procured in office-based workplaces can become particularly debilitating, since these categories of disorders principally cause distress to sensitive anatomical regions such as the neck, the lower back, but also the shoulders and the extremities of the upper limbs [15-19]. Symptoms can vary, but the occurrence of chronic pain and motor dysfunction are quite well-established [16]. The manifestations seem to be triggered either by isolated or combined micro traumas to muscles, tendons, joints, and nerves $[15,19]$. Often, the causes are attributed to poor habits in the workplace, such as repeated wrist movements (as in the act of dragging the mouse or clicking its buttons), and can also be the consequence of inappropriate posture and/or sitting in front of an office computer for extensive periods of time. It is thus important to take preventive measures, since work-related MSDs already represent a significant socio-economic burden in developed countries. According to a recent study by Van Eerd and coworkers [20], if no significant actions are undertaken by occupational health and safety practitioners, MSDs may become the leading cause of long absences from work and, even worse, cause permanent disabling effects. According to recent literature ([21,22], several workplace interventions have been implemented so far. Unfortunately, only a few studies have demonstrated that such measures had sustainable and quantifiable positive effects on symptoms and disability outcomes, although none have shown negative effects. Nonetheless, stretching exercises and resistance training programmes have emerged as highly-effective interventions to prevent office-based MSDs [23-25]. The general idea is that some programmes have the potential to be effective, but the scientific evidence is yet insufficient to provide the support needed in order to recommend their implementation in workplaces.

\subsection{The Potential Benefits of Use of Machine Learning Classification Algorithms in Musculoskeletal Pain Disorders}

\section{Highlight by Milos Ljubisavljevic}

Chronic musculoskeletal pain conditions, like fibromyalgia (FM), chronic muscle pain, chronic fatigue syndrome (CFS), etc. have a poor correlation between tissue abnormalities and clinical pain. This makes the diagnosis challenging, depending mostly on self-reporting and rather than objective findings. The last decade has witnessed significant progress in research regarding alterations 
of brain structure and function in chronic pain and musculoskeletal pain disorders. For example, when exposed to the same painful stimuli, patients with FM showed greater neurologic pain signature [26] responses. In spite of significant advances, the analysis of complex and multivariate brain imaging signals has proven challenging. Machine learning (ML), a data analytics method, is based on devising models and algorithms that lend themselves to prediction-making. It uses classifiers to identify to which set of categories an observation belongs (i.e., pain), by using a training set of data containing observations whose category membership is known. Recently several studies with promising results on neurophysiological and imaging signatures of musculoskeletal disorders using machine learning have appeared. Robinson et al. [27] used several ML algorithms to estimate different models representing brain volumes, mood ratings, and pain intensity ratings in FM patients and showed that although classification accuracy was reasonably high for brain volumes and pain intensity, models derived from self-report data outperformed neuroimaging models. A more recent study on FM patients showed the existence of a new pain-related classifier [28]. In FM patients, ML showed an augmented response in insula/operculum, which is related to sensory integration, and in medial prefrontal regions, which are related to self-reference. Also, they showed a reduced response in the lateral frontal cortex. Interestingly, a "multisensory" classifier, which was trained on non-painful stimulation, showed increased responses of the insula/operculum, posterior cingulate, and medial prefrontal regions and reduced responses of the primary and secondary sensory cortices, basal ganglia, and cerebellum [28]. Similarly, Boissoneault J et al. [29] described classification ML techniques which are capable of separating MRI-based brain biomarkers of chronic pain patients from healthy controls with high accuracy. Finally, Sevel et al. [30] used ML to compare the performance of structural MRI data and self-reported measures for distinguishing healthy from FM and CHS patients. Contrary to early reports (Robinson et al. 2015), they showed that MRI features perform better than self-reported measures. Furthermore, they identified structural abnormalities (hypertrophy and atrophy) in several brain regions, suggesting increased affective representation of pain in FM. In conclusion, it appears that ML classifiers analysis may provide new venues in distinguishing the distinct neural mechanisms in musculoskeletal disorders and potentially provide mechanistic information regarding their neural correlates.

Conflicts of Interest: The authors declare no conflict of interest.

\section{References}

1. Musumeci, G.; Aiello, F.C.; Szychlinska, M.A.; Di Rosa, M.; Castrogiovanni, P.; Mobasheri, A. Osteoarthritis in the XXIst century: Risk factors and behaviours that influence disease onset and progression. Int. J. Mol. Sci. 2015, 16, 6093-6112.

2. Keyserling, W.M. Workplace risk factors and occupational musculoskeletal disorders, Part 1: A review of biomechanical and psychophysical research on risk factors associated with low-back pain. AIHAJ 2000, 61, 39-50.

3. Gheno, R.; Cepparo, J.M.; Rosca, C.E.; Cotton, A. Musculoskeletal disorders in the elderly. J. Clin. Imaging Sci. 2012, 2, 39 .

4. Occhipinti, E.; Colombini, D. [Aging at work and musculoskeletal disorders]. Med. Lav. 2000, 91, 342-353.

5. Cross, M.; Smith, E.; Hoy, D.; Nolte, S.; Ackerman, I.; Fransen, M.; Bridgett, L.; Williams, S.; Guillemin, F.; Hill, C.L.; et al. The global burden of hip and knee osteoarthritis: Estimates from the global burden of disease 2010 study. Ann. Rheum. Dis. 2014, 73, 1323-1330.

6. Vitiello, V.; Germani, A.; Capuzzo Dolcetta, E.; Donini, L.M.; Del Balzo, V. The New Modern Mediterranean Diet Italian Pyramid. Ann. Ig. 2016, 28, 179-186.

7. Musumeci, G.; Trovato, F.M.; Pichler, K.; Weinberg, A.M.; Loreto, C.; Castrogiovanni, P. Extra-virgin olive oil diet and mild physical activity prevent cartilage degeneration in an osteoarthritis model: An in vivo and in vitro study on lubricin expression. J. Nutr. Biochem. 2013, 24, 2064-2075. 
8. Veronese, N.; Stubbs, B.; Noale, M.; Solmi, M.; Luchini, C.; Smith, T.O.; Cooper, C.; Guglielmi, G.; Reginster, J.Y.; Rizzoli, R.; et al. Adherence to a Mediterranean diet is associated with lower prevalence of osteoarthritis: Data from the osteoarthritis initiative. Clin. Nutr. 2016. [CrossRef]

9. Mihata, T.; Lee, T.Q.; Watanabe, C.; Fukunishi, K.; Ohue, M.; Tsujimura, T.; Kinoshita, M. Clinical results of arthroscopic superior capsule reconstruction for irreparable rotator cuff tears. Arthroscopy 2013, 29, 459-470.

10. Hirahara, A.M.; Adams, C.R. Arthroscopic superior capsular reconstruction for treatment of massive irreparable rotator cuff tears. Arthrosc. Tech. 2015, 4, e637-e641.

11. Petri, M.; Greenspoon, J.A.; Millett, P.J. Arthroscopic superior capsule reconstruction for irreparable rotator cuff tears. Arthrosc. Tech. 2015, 4, e751-e755.

12. Burkhart, S.S.; Denard, P.J.; Adams, C.R.; Brady, P.C.; Hartzler, R.U. Arthroscopic superior capsular reconstruction for massive irreparable rotator cuff repair. Arthrosc. Tech. 2016, 5, e1407-e1418.

13. Mihata, T.; McGarry, M.H.; Kahn, T.; Goldberg, I.; Neo, M.; Lee, T.Q. biomechanical role of capsular continuity in superior capsule reconstruction for irreparable tears of the supraspinatus tendon. Am. J. Sports Med. 2016, 44, 1423-1430.

14. Harensträm, A.; Staffan, M.; Bernston, E.; Bolin, M.; Ylander, J. Understanding the Organisational Impact on Working Conditions and Health; National Institute for Working Life: Stockholm, Sweden, 2006.

15. Aarås, A.; Horgen, G.; Ro, O. Work with the visual display unit: Health consequences. Int. J. Hum. Comput. Interact. 2000, 12, 107-134.

16. Kryger, A.I.; Andersen, J.H.; Lassen, C.F.; Brandt, L.P.A.; Vilstrup, I.; Overgaard, E.; Thomsen, J.F.; Mikkelsen, S. Does computer use pose an occupational hazard for forearm pain; from the NUTATA study. Occup. Environ. Med. 2003, 60, 11.

17. Hagberg, M.; Wegman, D.H. Prevalence rates and odds ratios of shoulder-neck disease in different occupational groups. Br. J. Ind. Med. 1987, 44, 602-610.

18. Bernard, B.P. Musculoskeletal Disorders and Workplace Factors: A Critical Review of Epidemiological Evidence for Work-Related Musculoskeletal Disorders of the Neck, Upper Extremity, and Low Back; Publication No 97-141; US Department of Health and Human Services: Cincinnati, OH, USA, 1997.

19. Ardahan, M.; Simsek, H. Analyzing musculoskeletal system discomforts and risk factors in computer-using office workers. Pak. J. Med. Sci. 2016, 32, 1425-1429.

20. Van Eerd, D.; Munhall, C.; Irvin, E.; Rempel, D.; Brewer, S.; van der Beek, A.J.; Dennerlein, J.T.; Tullar, J.; Skivington, K.; Pinion, C.; et al. Effectiveness of workplace interventions in the prevention of upper extremity musculoskeletal disorders and symptoms: An update of the evidence. Occup. Environ. Med. 2016, 73, 62-70.

21. Kennedy, C.A.; Amick, B.C., III; Dennerlein, J.T.; Brewer, S.; Catli, S.; Williams, R.; Serra, C.; Gerr, F.; Irvin, E.; Mahood, Q.; et al. Systematic review of the role of occupational health and safety interventions in the prevention of upper extremity musculoskeletal symptoms, signs, disorders, injuries, claims and lost time. J. Occup. Rehabil. 2010, 20, 127-162.

22. Tullar, J.M.; Brewer, S.; Amick, B.C., III; Irvin, E.; Mahood, Q.; Pompeii, L.A.; Wang, A.; Van Eerd, D.; Gimeno, D.; Evanoff, B. Occupational safety and health interventions to reduce musculoskeletal symptoms in the health care sector. J. Occup. Rehabil. 2010, 20, 199-219.

23. Mongini, F.; Evangelista, A.; Rota, E.; Ferrero, L.; Ugolini, A.; Ceccarelli, M.; Ciccone, G.; Galassi, C. Long-term benefits of an educational and physical program on headache, and neck and shoulder pain, in a working community. J. Pain 2009, 10, 1138-1145.

24. Andersen, C.H.; Andersen, L.L.; Gram, B.; Pedersen, M.T.; Mortensen, O.S.; Zebis, M.K.; Sjøgaard, G. Influence of frequency and duration of strength training for effective management of neck and shoulder pain: A randomised controlled trial. Br. J. Sports Med. 2012, 46, 1004-1010.

25. Sjogren, T.; Nissinen, K.J.; Jarvenpaa, S.K.; Ojanen, M.T.; Vanharanta, H.; Mälkiä, E.A. Effects of a workplace physical exercise intervention on the intensity of headache and neck and shoulder symptoms and upper extremity muscular strength of office workers: A cluster randomized controlled cross-over trial. Pain 2005, 116, 119-128.

26. Wager, T.D.; Atlas, L.Y.; Lindquist, M.A.; Roy, M.; Woo, C.W.; Kross, E. An fMRI-based neurologic signature of physical pain. N. Engl. J. Med. 2013, 368, 1388-1397.

27. Robinson, M.E.; O'Shea, A.M.; Craggs, J.G.; Price, D.D.; Letzen, J.E.; Staud, R. Comparison of machine classification algorithms for fibromyalgia: Neuroimages versus self-report. J. Pain 2015, 16, 472-477. 
28. López-Solà, M.; Woo, C.W.; Pujol, J.; Deus, J.; Harrison, B.J.; Monfort, J.; Wager, T.D. Towards a neurophysiological signature for fibromyalgia. Pain 2017, 158, 34-47.

29. Boissoneault, J.; Sevel, L.; Letzen, J.; Robinson, M.; Staud, R. Biomarkers for Musculoskeletal Pain Conditions: Use of Brain Imaging and Machine Learning. Curr. Rheumatol. Rep. 2017, 19, 5.

30. Sevel, L.; Letzen, J.; Boissoneault, J.; O'Shea, A.; Robinson, M.; Staud, R. MRI-based classification of chronic fatigue, fibromyalgia patients and healthy controls using machine learning algorithms: A comparison study. J. Pain 2016, 17, S60.

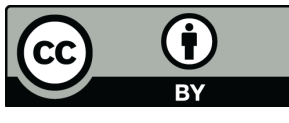

(c) 2017 by the authors. Licensee MDPI, Basel, Switzerland. This article is an open access article distributed under the terms and conditions of the Creative Commons Attribution (CC BY) license (http://creativecommons.org/licenses/by/4.0/). 